\title{
Genital ulcer disease in women in Durban, South Africa
}

\author{
N O'Farrell, A A Hoosen, K D Coetzee, J van den Ende
}

\begin{abstract}
Objective-To study the microbial aetiology of genital ulcer disease (GUD) in women.

Design-Microbial and clinical assessment of genital ulcers in women.

Setting-City Health sexually transmitted diseases clinic, King Edward VIII Hospital, Durban, South Africa.

Participants-100 Zulu women with genital ulceration who had not received antibiotics in the previous two weeks.

Results-Syphilis was diagnosed in $40 \%$, genital herpes in 18\%, donovanosis (granuloma inguinale) in $16 \%$, chancroid in $14 \%$, lymphogranuloma venereum in $7 \%$ and scabies in $2 \%$. No recognised cause was detected in $18 \%$. Secondary syphilis was diagnosed in $21 \%$, primary syphilis in $16 \%$ and mixed primary and secondary syphilis in 3\%. Multiple infections were detected in 13 women, of whom 12 had syphilis. Bleeding was observed from the ulcers of 59 during swab collection. Three women had HIV-1 antibodies. Neisseria gonorrhoeae was isolated from the ulcers and endocervix of two women and from the endocervix alone in nine. Generalised scabies was diagnosed in 14 .

Conclusions-All the major causes of GUD are prevalent in Zulu women in Durban: secondary syphilis was the commonest diagnosis. Donovanosis, which often presents late with large ulcers, and genital herpes are now significant problems. Mixed infections with coexisting syphilis are common. All women in this population with GUD should be treated for syphilis and receive oral antibiotics effective for chancroid and donovanosis.
\end{abstract}

Introduction

There are few clinics designated specifically for the

City Health STD Department, King Edward VIII Hospital, Durban

N O'Farrell

Department of Medical Microbiology, Faculty of Medicine, University of Natal, Durban, South Africa A A Hoosen, K D Coetzee, J van den Ende management of sexually transmitted diseases (STD) in Africa outside large cities. Elsewhere patients are seen routinely in general out-patient departments and are diagnosed on clinical grounds. Even in specialist centres the diagnosis of genital ulcer disease (GUD) must rely on initial clinical assessment in the absence of adequate rapid diagnostic techniques. Studies in African women from Nairobi ${ }^{1}$ and Johannesburg ${ }^{2}$ identified Haemophilus ducreyi as the most frequent cause of GUD and detected lower prevalences of syphilis. In Durban and Natal province, significant prevalences of positive syphilis serology were detected amongst antenatal clinic attenders without GUD. ${ }^{34}$ The recognition of congenital syphilis as an important cause of perinatal mortality locally further suggested syphilis was a cause of much unrecognised GUD. ${ }^{5}$ Previously donovanosis (granuloma inguinale) had received little mention in the medical literature from Africa. Following the introduction of a rapid staining technique for the detection of Donovan bodies, ${ }^{6}$ the numbers of male and female cases diagnosed increased immediately in Durban and suggested under-reporting of this condition.

Human immunodeficiency virus (HIV) infection is an escalating problem throughout southern Africa. GUD in seropositive women may promote the transmission of $\mathrm{HIV}^{7}$ and the control of GUD is likely to assume increasing importance in limiting the spread of HIV infection. A previous study in local men with GUD in 1984 demonstrated syphilis as the most common cause, ${ }^{8}$ but no similar study has been undertaken in women. In this study, we investigated the microbial aetiology of GUD amongst black women in Durban.

\section{Patients and methods}

One hundred consecutive Zulu women with GUD attending the City Health STD Clinic, King Edward VIII Hospital, Durban were entered into the study which was completed between August 1988 and January 1989.

Informed consent was obtained from all patients and written consent was obtained for HIV antibody testing. Women who had received antibiotics during the previous two weeks were excluded. A detailed history was obtained with the help of a clinic sister acting as interpreter. All women were examined in 
the lithotomy position and the size, site and nature of ulceration, presence of lymphadenopathy or skin rashes were recorded. A Cusco's speculum was passed to visualise the cervix except in women with extensive painful ulceration or those who were menstruating.

\section{Microbiological investigations}

Material for analysis was collected with cotton tipped swabs, applied to ulcers in the order of most likely clinical diagnosis. Dark field microscopic examination for Treponema pallidum was performed. Two media were used for the isolation of Haemophilus ducreyi: (1) gonococcal agar base (GIBCO) with $1 \%$ bovine haemoglobin, $5 \%$ fetal calf serum supplemented with $1 \%$ isovitalex (BBL) and vancomycin $5 \mathrm{mg} / \mathrm{l}$, (2) Muller-Hinton (BBL) agar plates with $5 \%$ sterile horse blood heated to $75{ }^{\circ} \mathrm{C}$, $1 \%$ isovitalex (BBL) and vancomycin $5 \mathrm{mg} / \mathrm{l}$. Procedures for the isolation and identification of $H$ ducreyi were as previously described. ${ }^{8}$ Slides were stained by the RapiDiff technique (CSD, Booysens 2016, SA) for the detection of Donovan bodies characteristic of donovanosis. Lymphogranuloma venereum (LGV) was diagnosed by examination of ulcer material using direct immunofluorescence (Microtrak, SYVA, UK). (Neither chlamydial culture nor serology (microimmunofluorescence) were available.) One swab was placed in viral transport medium and kept on ice until transferred to the laboratory. Specimens were inoculated on human lung fibroblast cells or primary Vervet monkey kidney cells incubated at $37^{\circ} \mathrm{C}$ and Herpes simplex virus (HSV) identified and confirmed as described previously. ${ }^{8}$ A swab specimen was inoculated on modified New York City (MNYC) medium for the isolation of Neisseria gonorrhoeae. Swab specimens from the endocervix were used for the preparation of a smear for Gram staining and inoculated onto MNYC for the isolation of $N$ gonorrhoeae. An endocervical Papanicoloau (PAP) smear was also prepared.

The following serological tests for syphilis were carried out: (1) rapid plasma reagin (RPR) test (BBL). (2) $T$ pallidum haemagglutination assay (TPHA) (FURJIREBIO). (3) Fluorescent treponemal antibody absorption (FTA-ABS) tests for IgG and IgM (Wellcome Diagnostics). The criteria chosen for the diagnosis of syphilis were either positive dark field microscopy or a positive FTA IgM test or both. HIV antibody testing was performed using a recombinant HIV-1 ELISA kit (Abbott Diagnostics) and positive sera confirmed by using the DuPont Western Blot (Biotech Research Laboratories). Specimens were considered positive if antibodies to $\mathrm{p} 24$ and either gp41 or gp120/160 were detected.
Table 1 Duration of symptoms before presentation in 100 women with genital ulceration

\begin{tabular}{ll}
\hline Duration of symptoms (days) & No $(\%)$ of women $(N=100)$ \\
\hline $1-7$ & 47 \\
$8-14$ & 17 \\
$15-21$ & 13 \\
$22-30$ & 10 \\
$31-90$ & 7 \\
$91-365$ & 2 \\
$>365$ & 4 \\
\hline
\end{tabular}

\section{Results}

The mean age of the women was 21.9 (range 14-48) years. Ninety-two were unmarried and eighty-five were new attenders to the clinic. Prior consultations with other agencies for their present clinical problem were sought by six women from traditional healers (inyangas), three from private doctors, three from nurses and two from hospital doctors. Five women did not complain of ulcers and three were unaware of their presence. Eight women were pregnant. One woman developed ulcers following rape and one denied previous penetrative vaginal intercourse. The duration of symptoms ranged from two days to one and half years (table 1). Ulcers had been present for more than one year in four women.

The mean number of ulcers was $4 \cdot 8$ (range 1 to 40 ). Twenty-eight women had one ulcer, 14 had two ulcers, 30 had three to five ulcers, 14 had six to ten ulcers and 13 had more than ten ulcers. The diameter

Table 2 Sexually transmitted diseases and other infections in 100 Zulu women with genital ulcers

No $(\%)$ of women

\begin{tabular}{lc}
\hline Single infections: & 28 \\
Syphilis & 11 \\
$\quad$ Pimary & 15 \\
$\quad$ Secondary & 2 \\
Primary + secondary & 12 \\
Genital herpes & 12 \\
Chancroid & 10 \\
Donovanosis & 5 \\
LGV & \\
Double infections: & 4 \\
Syphilis + genital herpes & 3 \\
Syphilis + donovanosis & 2 \\
Syphilis + chancroid & 1 \\
Syphilis + LGV & 1 \\
Donovanosis + genital herpes & \\
Triple infections: & 1 \\
Syphilis + genital herpes + donovanosis & 1 \\
Syphilis + LGV + donovanosis & 20 \\
None of above detected & \\
Additional diagnoses: & \\
Gonorrhoea & \\
$\quad$ Uervix alone & 9 \\
Scabies + cervix & 2 \\
& 16 (sole cause of \\
HIV-1 seropositive & in 2 women) \\
Hition & 3
\end{tabular}


Table 3 Features of genital ulcer disease in women

\begin{tabular}{|c|c|c|c|c|}
\hline Feature & $\begin{array}{l}\text { Primary syphilis } \\
(n=11)\end{array}$ & $\begin{array}{l}\text { Secondary syphilis } \\
(n=15)\end{array}$ & $\begin{array}{l}\text { Genital herpes } \\
(n=12)\end{array}$ & $\begin{array}{l}\text { Chancroid } \\
(n=12)\end{array}$ \\
\hline $\begin{array}{l}\text { Mean number of ulcers } \\
\text { Range } \\
\text { Mean diameter of largest ulcer (mms) } \\
\text { Range } \\
\text { Mean delay before presentation (days) } \\
\text { Range } \\
\text { Bleeding from ulcer site during swab collection } \\
\text { Inguinal lymphodenopathy: } \\
\text { Unilateral }(n=19) \\
\text { Bilateral }(n=36) \\
\text { BUBO }(n=4)\end{array}$ & $\begin{array}{l}2 \cdot 0 \\
(1-3) \\
10 \cdot 6 \\
(5-30) \\
11 \cdot 5 \\
(6-30) \\
8\end{array}$ & $\begin{array}{l}12 \cdot 5 \\
(3-20) \\
7 \cdot 4 \\
(3-30) \\
27 \cdot 5 \\
(2-60) \\
6\end{array}$ & $\begin{array}{l}2 \cdot 6 \\
(1-7) \\
5 \cdot 0 \\
(3-7) \\
10 \cdot 6 \\
(2-56) \\
3\end{array}$ & $\begin{array}{l}2 \cdot 9 \\
(1-5) \\
8 \cdot 3 \\
(5-15) \\
12 \cdot 8 \\
(1-21) \\
10\end{array}$ \\
\hline
\end{tabular}

of the largest ulcer ranged from three $\mathrm{mm}$ to $50 \mathrm{~mm}$. Ulcers were situated inside the vagina or at the introitus in 33 women, extra-vaginally in 49 and in both areas in 18. Bleeding from the ulcer site was observed in 59 women during swab collection. Cervical erosions were detected clinically in ten women. Bilateral inguinal lymphadenopathy was present in 36 women and unilateral lymphadenopathy in 19. Four women had buboes, one requiring aspiration. Three women had large areas of ulceration in the inguinal region following spontaneous rupture of buboes.

Forty women, including three with mixed primary and secondary lesions, were diagnosed with active syphilis (table 2). Ulcerated papules and plaques of condylomata lata were observed in 24 women and primary chancres in 19. Mixed infections were detected in 13 women, of whom 12 had syphilis. Ulcers of primary syphilis tended to be of intermediate diameter (table 3), occurring either singly or in pairs, which bled during swab collection and were situated both intra- and extra-vaginally. Secondary syphilitic lesions were multiple, associated with a long delay before presentation and situated extravaginally.

Genital herpes was detected in 12 women as a single infection, as a mixed double infection with syphilis in four and donovanosis in one and as a mixed triple infection with syphilis and donovanosis in one. HSV ulcers tended to be fewer and of the smallest diameter. Bleeding was observed in three of 13 during specimen collection. HSV infection of the cervix was detected on Papanicoloau smears in two women, one with vaginal herpetic lesions and one without vulval sores.

Chancroid was diagnosed (by the isolation of $H$ ducreyi) as a single infection in 12 women and as a mixed infection with syphilis in two others. Bleeding from the ulcer site was observed in 10 of the 12 women with chancroid alone during swab collection.

Donovanosis, diagnosed by the presence of Donovan bodies, was demonstrated in 10 women as a single infection, four of whom were pregnant. Mixed infections with donovanosis were detected in six women (table 2). The mean diameter of donovanosis ulcers was the largest. Bleeding was noted during swab collection in eight of the 10 patients with single infections. The mean delay before presentation was 165 (range 7-540) days. Donovan bodies were detected in all four women with ulceration present longer than one year. Ulcers were either intravaginal or adjacent to the vagina in seven of the 10 women with single infection and three of the six with mixed infections. Ulcers were observed both inside the vagina and in the inguinal region in three women with single infections. Hypertrophic lesions were present in three women.

LGV was diagnosed as a single infection in five women and in two as a mixed infection. Bleeding was observed in four of five single infections during swab collection. All LGV ulcers were small, intravaginal and deeper than ulcers of $\mathrm{HSV}$ or chancroid. $N$ gonorrhoeae was isolated from the ulcers and endocervices of two women and from the endocervix alone in nine women.

In 20, none of the above causes of GUD was demonstrated. Scabies was considered to be the most likely diagnosis in two women with extensive multiple vulval ulceration, one of whom denied sexual intercourse. Syphilis serology tests were positive in nine of these 20 . One woman from a rural area had extensive vulval ulceration suggestive clinically of vulval carcinoma but was lost to follow up. The 11 remaining patients with negative serological tests for syphilis were diagnosed clinically either as herpes genitalis (six) and chancroid (five). These ulcers tended to be small and to bleed during swab collection. Three of the 92 women who consented to HIV antibody tests were seropositive.

Inflammatory changes were reported on PAP smears in 34 of $73(46.6 \%)$, cervical intraepithelial neoplasia in four $(5.5 \%)$, human papillomavirus in seven $(9.6 \%)$ and infections with Trichomonas vaginalis in $25(34 \cdot 2 \%)$ and HSV in two $(2 \cdot 7 \%)$. Bleeding was noted in nine of 73 , of whom six had inflammatory changes detected. More noticeable bleeding 


\begin{tabular}{|c|c|c|c|c|c|}
\hline $\begin{array}{l}\text { Donovanosis } \\
(n=10)\end{array}$ & $\begin{array}{l}L G V \\
(n=5)\end{array}$ & $\begin{array}{l}\text { No cause } \\
(n=18)\end{array}$ & $\begin{array}{l}\text { Mixed infections } \\
(n=13)\end{array}$ & $\begin{array}{l}\text { Primary + secondary } \\
\text { syphilis }(n=2)\end{array}$ & $\begin{array}{l}\text { Scabies } \\
(n=2)\end{array}$ \\
\hline $\begin{array}{l}1 \cdot 8 \\
(1-3) \\
28 \cdot 3 \\
(10-50) \\
165 \\
(7-540) \\
8\end{array}$ & $\begin{array}{l}2 \cdot 2 \\
(1-3) \\
12 \cdot 0 \\
(5-20) \\
9 \cdot 5 \\
(4-21) \\
3\end{array}$ & $\begin{array}{l}3 \cdot 3 \\
(1-25) \\
7 \cdot 8 \\
(2-20) \\
10 \cdot 3 \\
(3-60) \\
12\end{array}$ & $\begin{array}{l}4 \cdot 7 \\
(1-10) \\
12 \cdot 6 \\
(10-50) \\
17 \cdot 5 \\
(3-42) \\
6\end{array}$ & & \\
\hline $\begin{array}{l}1 \\
1\end{array}$ & $\begin{array}{l}1 \\
2 \\
1\end{array}$ & $\begin{array}{l}5 \\
7 \\
2\end{array}$ & $\begin{array}{l}3 \\
6 \\
1\end{array}$ & $\begin{array}{l}1 \\
1\end{array}$ & - \\
\hline
\end{tabular}

was observed in one woman with Schistosoma haematobium infection of the cervix detected on a PAP smear.

\section{Discussion}

This study has found that all the major sexually transmitted causes of GUD are prevalent amongst black women in Durban, South Africa. Syphilis was the most common cause of GUD in $40 \%$, in keeping with a similar current prevalence of $42 \%$ in local men. ${ }^{9}$ Clinical signs of secondary syphilis were more common than primary syphilis suggesting that women with primary lesions either experience asymptomatic ulcers within the vagina or disregard their symptoms. Well defined criteria were set for the diagnosis of syphilis. Although a positive RPR dilution of $1: 2$ has been suggested as indicating active infection, ${ }^{10}$ such a criterion if applied to our study population with a high background seropositivity rate for syphilis would have overestimated the numbers of true cases by including infections treated previously.

Genital herpes has been regarded largely as a disease of Western society but was found to be a significant cause of GUD in this study. The prevalence of $18 \%$ is comparable with the $20 \%$ recorded in Rwanda, ${ }^{11}$ but is higher than the reported prevalences of $2 \%$ in Nairobi ${ }^{1}$ and $6 \%$ in Johannesburg. ${ }^{2}$ In South Africa, HSV infection is not classified as a STD for reporting purposes under the national STD coding system and therefore may have gone unrecognised previously or been misdiagnosed as chancroid, which shares many similar clinical features.

Haemophilus ducreyi was isolated from $14 \%$ of women in our study, yielding a much lower prevalence of proven chancroid than found in Nairobi $(48 \%)^{1}$ and Johannesburg $\left.(46 \%)\right)^{2}$ Some studies in which high prevalence of chancroid have been detected have included significant numbers of prostitutes and their clients. In South Africa prostitution is illegal and prostitutes are rarely identified in this clinic. When compared to the ulcers caused by HSV, chancroid were of slightly larger diameter and tended to bleed during swab application. The presence of lymphadenopathy did not assist in distinguishing between the two infections.

Donovanosis was diagnosed in 16 women by the detection of Donovan bodies in stained smears. Following the introduction of a rapid tissue smear test the need for either punch or crushed biopsy specimens was foregone in this clinic. All Donovan body positive ulcers were larger than $10 \mathrm{~mm}$ in diameter and biopsy may be required to confirm the diagnosis in smaller lesions. Donovan bodies were detected in four of eight pregnant women, of whom three had hypertrophic lesions. More extensive ulcers are described during pregnancy ${ }^{12}$ and may reflect altered maternal immunocompetence. LGV was detected in $7 \%$ of women in our study and was the most likely clinical diagnosis in two other women. The presence of lymphadenopathy in four of the five women with single LGV infections made contamination with chlamydial cervical infection unlikely. The smaller diameter of ulcers in our patients contrasted with the larger ulcers from which Chlamydia trachomatis was isolated in a Swaziland study. ${ }^{13}$

PAP smears are not performed routinely in STD clinics in South Africa although provision for the service is made by the government. The significant proportion of women with cervical inflammatory changes in our study may reflect the high prevalences of vaginal pathogens detected locally, particularly Trichomonas vaginalis. ${ }^{4}$ In examining the risk of $\mathrm{HIV}$ transmission in women with GUD, covert cytological abnormalities following present or past STD's may act as cofactors in facilitating HIV infection ${ }^{14}$ and should be taken into account.

The majority of ulcers for which no diagnosis was made were of similar appearances to genital herpes or chancroid, that is, small ulcers in groups of three or four. Four women had lesions typical of syphilis but were excluded from the diagnosis by our strict criteria despite strongly positive TPHA, RPR and FTA-ABS tests. Competitive inhibition of the FTAIgM reaction by high levels of IgG is a possible 
explanation in these cases and the true prevalence of active syphilis was probably underestimated in this study.

Mixed infections were detected in 13 women, of whom 12 had syphilis. Coexisting infections make clinical diagnosis unreliable and treatment for syphilis is essential. Cotrimoxazole or erythromycin should also be used given their efficacy in most cases of chancroid, donovanosis and secondary infections associated with HSV ulceration. Recent emergence of high-level sulphonamide and trimethoprim resistance in Kenyan isolates of $H$ ducreyi ${ }^{15}$ is a cause for concern and studies on the antimicrobial sensitivities of local isolates are required.

Antibodies to HIV-1 were detected in three women. HIV infection is being increasingly detected in black heterosexual women attending STD clinics in South Africa. ${ }^{16}$ Locally young women in the 15-19 age group and those with genital herpes or a past history of syphilis are at increased risk of HIV infection. ${ }^{14}$ Increased medical awareness of the importance of GUD could slow the spread of HIV infection in this Zulu population who are now the group at highest risk of infection in South Africa. ${ }^{17}$

We thank the staff at the City Health STD Clinic, including all doctors, sisters and health assistants, especially Bernard Zulu, for their co-operation.

Address for correspondence: Dr Nigel O'Farrell, Lydia Department, St Thomas' Hospital, London SE1 7RH, UK.
1 Plummer FA, D'Costa LJ, Nsanze H, et al. Clinical and microbiological studies of genital ulcers in Kenyan women. Sex Transm Dis 1985;12:193-7.

2 Duncan MO, Ballard RC, Bilgeri YR, Fehler HG. Sexually acquired genital ulcerations in urban black women. Southern African Journal of Sexually Transmitted Diseases 1984;4:23-5.

3 Hoosen AA, Ross SM, Mulla MJ, Patel M. The incidence of selected vaginal infections among pregnant urban blacks. $S$ Afr Med J 1981;59:827-9.

4 O'Farrell N, Hoosen AA, Kharsany AB, van den Ende J. Sexually transmitted pathogens in pregnant women in a rural South African community. Genitourin Med 1989;65:276-80.

5 Ross SM, MacPherson T, Wallace J, Khatree MH, Naeye RL, Appelbaum PC. Unsuccessful pregnancies-report on 200 perinatal postmortems. S Afr Med J 1978;54:828-9.

6 O'Farrell N, Hoosen AA, Coetzee K, van den Ende J. A rapid stain for the diagnosis of granuloma inguinale (donovanosis). Genitourin Med 1990;66:200-1.

7 Kreiss JK, Coombs R, Plummer F, et al. Isolation of human immunodeficiency virus from genital ulcers in Nairobi prostitutes. J Infect Dis 1989;160:380-4.

8 Coovadia YM, Kharsany AB, Hoosen AA. The microbial aetiology of genital ulcers in black men in Durban, South Africa. Genitourin Med 1985;61:266-9.

9 O'Farrell N, Hoosen AA, Coetzee K, van den Ende J. Genital ulcer disease in men in Durban, South Africa. 35th General Assembly of the International Union Against the Venereal Diseases and the Treponematoses, London, May 1990.

10 Ursi JP, Van Dyck E, Van Houtte C. Syphilis in Swaziland. A serological survey. Br $J$ Venereal Dis 1981;57:95-9.

11 Bogaerts J, Ricart CA, Van Dyck E, Piot P. The etiology of genital ulceration in Rwanda. Sex Transm Dis 1989;16:123-6.

12 Latif AS, Mason PR, Paraiwa E. The treatment of donovanosis (granuloma inguinale). Sex Transm Dis 1988;15:27-9.

13 Meheus A, Van Dyck E, Ursi JP, Ballard RC, Piot P. Etiology of genital ulceration in Swaziland. Sex Transm Dis 1983;10:33-5.

14 O'Farrell N, Windsor I, Becker P. Risk factor for HIV-1 amongst STD clinic attenders in Durban, South Africa. Sixth International Conference on AIDS San Francisco, June 1990. (Abstract F.C. 604.)

15 Jessamine PG, Ronald AR. Chancroid and the role of genital ulcer disease in the spread of human retroviruses. Med Clin North Am 1990;74:1417-31.

16 O'Farrell N, Windsor I. Prevalence of HIV antibody in recurrent attenders at a sexually transmitted disease clinic. $S$ Afr Med J 1988;74:104-5.

17 Schoub BD, Smith AN, Lyons SF, et al. Epidemiological considerations of the present status and future growth of the acquired immunodeficiency syndrome epidemic in South Africa. $S$ Afr Med J 1988;74:153-7. 